\title{
Final-state interaction in Compton scattering from electron liquids
}

\author{
C. Sternemann, ${ }^{1}$ K. Hämäläinen, ${ }^{2}$ A. Kaprolat, ${ }^{1}$ A. Soininen, ${ }^{2}$ G. Döring, ${ }^{1}$ C.-C. Kao, ${ }^{3}$ S. Manninen, ${ }^{2}$ and W. Schülke \\ ${ }^{1}$ Institute of Physics, University of Dortmund, D-44221 Dortmund, Germany \\ ${ }^{2}$ Department of Physics, POB 9, FIN-00014 University of Helsinki, Finland \\ ${ }^{3}$ National Synchrotron Light Source, Brookhaven National Laboratory, Upton, New York 11973
}

(Received 14 July 2000)

\begin{abstract}
We present Compton profiles (CP's) of Li measured with momentum space resolution of 0.02 a.u. and 8-9 $\mathrm{keV}$ incident photon energy. The valence CP's are found to be asymmetric in shape and the predicted sharp features at the Fermi break are drastically smeared out. The observed additional broadening of the CP beyond experimental resolution is predominantly attributed to the finite width of the spectral density function of the excited particle. The asymmetry agrees well with calculations using a many-particle scheme, which will be useful for interpreting final states in soft x-ray and photoemission.
\end{abstract}

Inelastic x-ray scattering spectroscopy (IXSS), when performed within a regime of momentum transfer $\mathbf{q}$ and energy transfer $\hbar \omega$, where the so-called impulse approximation (IA) is assumed to be valid (Compton regime), yields information on fundamental ground state properties of the scattering electron system, namely on the projection of the electron momentum density (EMD) on q referred to as Compton profile (CP). ${ }^{1}$ The application of high intensity synchrotron radiation especially makes feasible a momentum space resolution $\Delta p_{z}$ down to a few percent of the Fermi momentum $\left(p_{F}\right)$ of simple metals, thus opening a unique possibility to investigate experimentally correlation-induced features of the EMD near $p_{F}$. Nevertheless, such a high momentum space resolution together with an appropriate statistical accuracy has been achieved until now only with incident $\mathrm{x}$-ray photon energies around $10 \mathrm{keV} .^{2}$ At those relatively low energies the validity of the IA becomes a potential problem, since the application of the IA requires, crudely speaking, that the transferred energy should be much larger than the characteristic energies of the scattering system (binding energy for the core and Fermi energy for the valence electrons). ${ }^{3}$ Indeed, deviations from the IA have been found, for instance, in the $1 s$ core contribution of CP's of graphite measured with an incident photon energy of $12.8 \mathrm{keV} .{ }^{4}$ A lot of theoretical effort has been brought into play to describe these deviations properly. ${ }^{5}$ On the other hand, based on an early work of Platzman and Tzoar, ${ }^{6}$ it was generally assumed so far that the $\mathrm{CP}$ of a nearly free electron system measured at $10 \mathrm{keV}$ can fully be interpreted in terms of the IA, so that it represents the valence EMD. However, it has been pointed out by Schülke et al. ${ }^{7}$ that, even under the above conditions, finalstate interaction effects on the $\mathrm{CP}$ must be taken into account. Therefore, it seems to be urgently requested to investigate more systematically how a deviation from the prerequisits of the IA may influence a CP measurement along with the information about EMD drawn from it. This is more important since significant discrepancies between the local density approximation (LDA) (Ref. 8) and quantum Monte Carlo CP calculations ${ }^{9}$ on the one hand and experimental CP's of $\mathrm{Li}^{7,8}$ have been found as far as the trace of the Fermi break is concerned.
We have found strong indications of final-state interactions in the experimental $\mathrm{CP}$ data of Li metal measured with incident photon energies somewhat below $10 \mathrm{keV}$, so that we have to conclude that the prerequisits for the IA are not fulfilled for CP experiments on valence electrons in the corresponding range of energy and momentum transfer. In detail, the CP's exhibit an additional broadening which could clearly be separated from the experimental broadening due to the finite momentum space resolution. The features of the $\mathrm{CP}$ at $p_{F}$, which have to be attributed to the finite step of the occupation number density, are still smeared out, especially since no sharp Fermi break can be resolved. This broadening will be interpreted as a convolution of the $\mathrm{CP}$ with the spectral density function (SDF) of the excited particle. Moreover, we found the asymmetry of the CP's to be correctly predicted by the first and second order vertex correction. This asymmetry could clearly be separated from an asymmetry caused by the deviations from the IA of the core $(1 s)$ contributions. Based on our many-particle calculation scheme (jellium $G W$ approximation) we interpret this asymmetry as an indication of an incomplete cancellation of the self-energy (SE) correction and the vertex correction. The good agreement between experiment and these calculations in this particular case encourages their extension to a wider range of $q$ in IXSS and their application to the interpretation of finalstate effects in other excitation studies.

The relation between the double differential scattering cross section and the imaginary part of the total electron polarization function $\chi(\mathbf{q}, \omega)$ (Ref. 10) is given by

$$
\begin{aligned}
\frac{d^{2} \sigma}{d \Omega d \omega} & \sim-\frac{1}{\pi} \operatorname{Im} \chi(\mathbf{q}, \omega) \\
& =\sum_{f} \mid\left\langle f\left|\sum_{j} e^{i \mathbf{q} \cdot \mathbf{r}_{\mathbf{j}}|i\rangle}\right|^{2} \delta\left(\hbar \omega-E_{f}+E_{i}\right),\right.
\end{aligned}
$$

where the $j$ sum is over the electrons with position $\mathbf{r}_{j}$. As pointed out by Eisenberger and Platzman, ${ }^{3}$ the essence of the IA consists in neglecting the influence of the potential energy part of the total system Hamiltonian on the energy difference in Eq. (2) between the energy $E_{i}$ of the many-particle ground state $|i\rangle$ and the energy $E_{f}$ of the final states $|f\rangle$ in the scat- 
a)

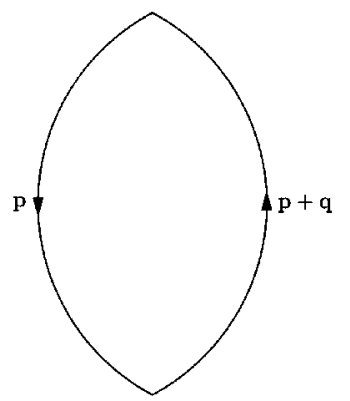

b)

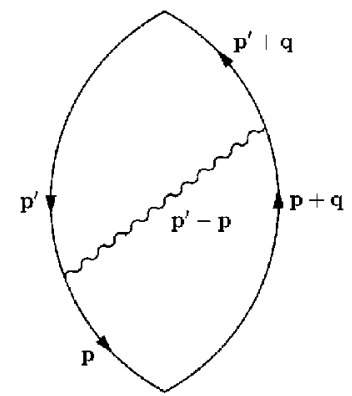

c)

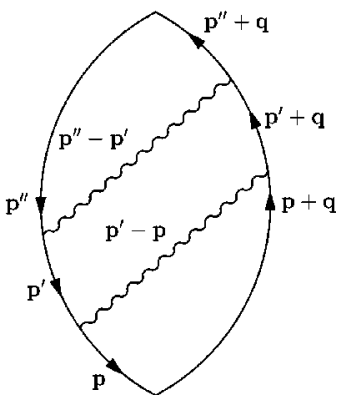

FIG. 1. Calculated Feynman graphs, for details see the text. tering process, which means $\delta\left(\hbar \omega-E_{f}+E_{i}\right) \rightarrow \delta[\hbar \omega-(\mathbf{p}$ $\left.+\mathbf{q})^{2} / 2+\mathbf{p}^{2} / 2\right]$, and $\left|\left\langle f\left|\Sigma_{j} \exp \left(i \mathbf{q} \cdot \mathbf{r}_{\mathbf{j}}\right)\right| i\right\rangle\right|^{2} \rightarrow|\langle\mathbf{p} \mid i\rangle|^{2}=\rho(\mathbf{p})$, thus ending up with

$$
\frac{d^{2} \sigma}{d \Omega d \omega} \sim J(\mathbf{p} \cdot \mathbf{q} / q) \sim \int d \mathbf{p} \rho(\mathbf{p}) \delta\left(\hbar \omega-\mathbf{q}^{2} / 2-\mathbf{p} \cdot \mathbf{q}\right)
$$

where $J\left(p_{z}\right)$ with $p_{z}=\mathbf{p} \cdot \mathbf{q} / q$ is the CP and $\rho(\mathbf{p})$ the EMD.

In terms of the jellium model, this interpretation of the IA means that we have to neglect all final-state interactions, more precisely, to neglect both all interactions of the excited particle with the many-particle system mediated by the polarization of this medium due to the existence of the excited particle (theoretically described as SE correction to the freeparticle polarizability) and the interaction of the excited particle with the hole left behind (particle-hole vertex correction to the free-particle polarizability). SE correction would cause, due to the imaginary part of the SE, an additional broadening of the imaginary part of the polarizability and therefore, an additional broadening of the CP. Moreover, due to the combined effect of the real and imaginary part of the $\mathrm{SE}$, the CP both can be shifted on the $p_{z}$ scale relative to its pure kinematical (IA) position and can become asymmetrically shaped with respect to its IA shape, which is symmetrical to $p_{z}=0$. The vertex correction, which is known to cancel to a certain extent the SE correction to the polarizability, ${ }^{11}$ will also cause an asymmetry of the profiles but opposite to the asymmetry produced by the SE correction. Thus the fingerprints of final-state effects one has to be aware of are the following: additional broadening of the $\mathrm{CP}$, shift of the $\mathrm{CP}$ on the $p_{z}$ scale, and asymmetric shape.

In order to exclude possible systematic experimental uncertainties two fully independent experiments were performed, one at the beamline $G 3$ of DESY/HASYLAB and the other at the beamline $X 21$ of NSLS. The radiation was monochromatized utilizing a Ge311 double crystal monochromator (single reflection bent triangular Si220 crystal at NSLS) and the scattered photons were analyzed within Rowland circle geometry using a spherically bent Ge800 (Si444) analyzer crystal. Both measurements were accomplished using the so-called inverse geometry, i.e., scanning the incident energy and keeping the analyzer energy fixed at $8.789 \mathrm{keV}$ $(7.921 \mathrm{keV})$ corresponding to a Bragg angle of $86^{\circ}\left(86.8^{\circ}\right)$. Directional CP's were measured with scattering vector $\mathbf{q} \|$ [100], [110], [111], and [311] at a scattering angle of $164^{\circ}$ $\left(160^{\circ}\right)$ with an overall energy resolution of $2.3 \mathrm{eV}(1.8 \mathrm{eV})$. The total momentum space resolution taking into account the uncertainty of the scattering angle due to the finite size of the analyzer crystal was 0.02 a.u. (0.03 a.u.) at the CP maxi- mum. More detailed descriptions of the experimental setup can be found elsewhere. ${ }^{12,13}$ The Li single crystal was a cuboid with $6.5 \mathrm{~mm}$ side length and $9 \mathrm{~mm}$ height for the HASYLAB experiment. At NSLS three identical discs with diameter of $20 \mathrm{~mm}$ and a thickness of $4 \mathrm{~mm}$ were cut having the surface normal along directions of [100], [110], and [111]. All samples were etched just before the measurements and fixed into a vacuum chamber to avoid oxidation. For each direction several single spectra were measured. After energy dependent corrections ${ }^{7}$ the data were converted to momentum scale and normalized to theoretical CP's. The $p_{z}=0$ position could be fixed to better than \pm 0.005 a.u. The multiple scattering contribution to the $\mathrm{CP}$ was calculated and found to have no significant influence on the presented results.

Within the range of energy and momentum transfer under consideration, $\chi(\mathbf{q}, \omega)$ of Eq. (1) can be replaced by the proper polarization function (PPF) $\chi_{S C}(\mathbf{q}, \omega) .{ }^{10}$ Within the limits of the jellium model we have calculated the PPF according to the graphical representation in Fig. 1(a), where the solid lines mean either the free-particle Greens function thus obtaining $\chi_{S C}^{0}(\mathbf{q}, \omega)$, the well known RPA (Lindhardt) result, or the fully dressed Greens function, ending up with $\chi_{S C}^{S E}(\mathbf{q}, \omega)$, which by using self-energies within the standard GW approximation and by screening the Coulomb potential by the RPA dielectric function ${ }^{14}$ contains the SE correction of $\chi_{S C}^{0}(\mathbf{q}, \omega)$ to all orders of the screened Coulomb potential, ${ }^{15}$ so that its imaginary part reads

$$
\begin{aligned}
\operatorname{Im}_{\chi_{S C}^{S E}(\mathbf{q}, \omega)=} & -\int \frac{d^{3} p}{(2 \pi)^{3}} \int_{-\omega+E_{F}}^{E_{F}} \frac{d E}{2 \pi} A(\mathbf{p}, E) \\
& \times A\left(\mathbf{p}+\mathbf{q}, E+\omega-\omega_{s}\right),
\end{aligned}
$$

where $A(\mathbf{p}, E)$ is the SDF, ${ }^{16}$ and the adjustable parameter $\omega_{s}$ takes into account approximately deviations of the excitation energies from the jellium model induced by the lattice potential.

Therefore the special shape of both, the hole-SDF with its well-known two-peak structure (plasmaron and quasiparticle peak), ${ }^{16}$ and the particle SDF with its asymmetrical shape and finite width are influencing the PPF according to the convolution expressed in Eq. (4). Especially, if we neglect $\omega_{s}$ together with the real part of the on-shell SE corresponding to $\mathbf{p}+\mathbf{q}$ in Eq.(4) and replace $\mathrm{E}$ in the particle SDF by $\epsilon^{0}(p)$, the free-particle energy, one ends up with

$$
\operatorname{Im} \chi_{S C}^{S E}(\mathbf{q}, \omega)=-\int \frac{d^{3} p}{(2 \pi)^{3}} \rho(\mathbf{p}) A\left(\mathbf{p}+\mathbf{q}, \epsilon^{0}(p)+\omega\right)
$$




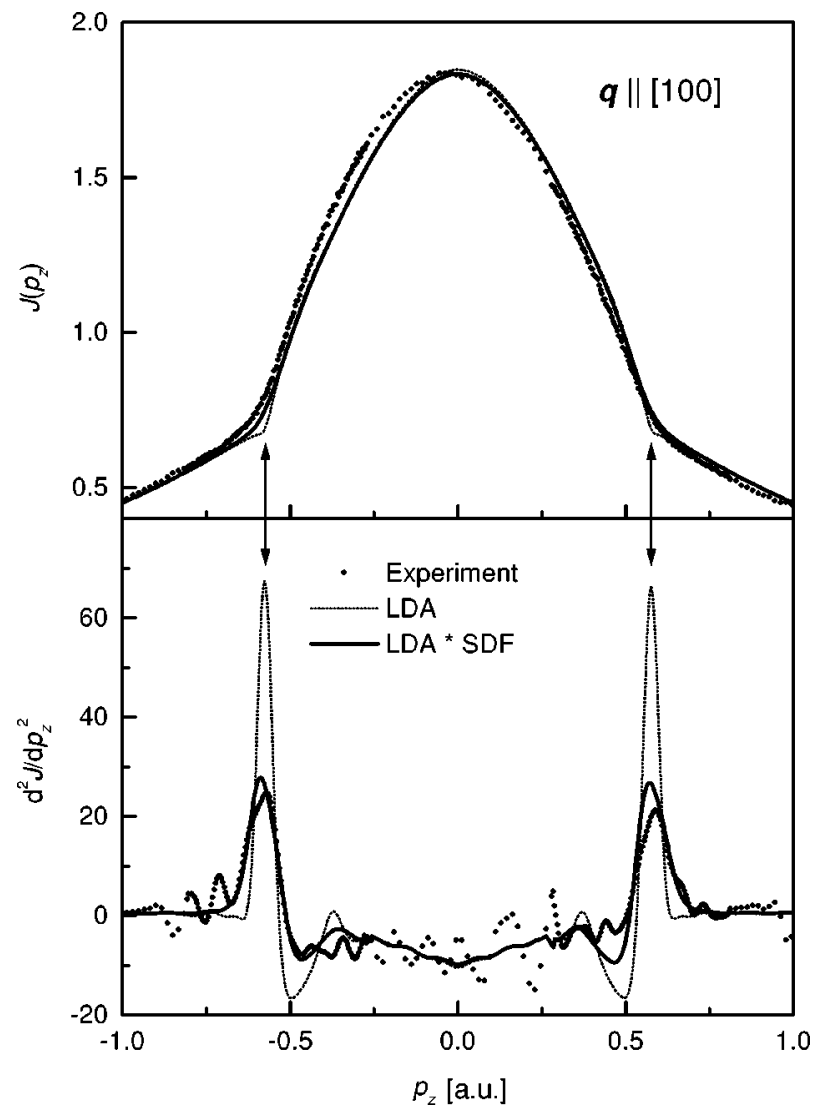

FIG. 2. CP's of Li and the corresponding second derivatives. Experiment compared to LDA theory ${ }^{8}$ and LDA theory convoluted with the particle SDF according to Eq. (5). All calculations include the experimental resolution. The arrows indicate the LDA Fermi momentum ( $p_{F}=0.576$ a.u.).

$$
\rho(\mathbf{p})=\int_{-\infty}^{E_{F}} \frac{d E}{2 \pi} A(\mathbf{p}, E)
$$

which tells us that one ingredient of the SE correction to the $\mathrm{CP}$, built up by the fully correlated $\operatorname{EMD}[\rho(\mathbf{p})$ of Eq. (6)], consists in convoluting the $\mathrm{CP}$ with the particle SDF, so that the resulting smearing of the Fermi break must be considered as a unique fingerprint of the interaction of the excited electron with the rest of the system.

Finally we have added the particle-hole vertex correction, first and second order ${ }^{17}$ in the screened Coulomb potential, to the fully SE corrected PPF by calculating the graphs of Figs. $1(b)$ and $1(c)$ and those with time reversed inner lines, where the Coulomb interaction between particle and hole is dynamically screened by the RPA dielectric function.

The experimental CP of $\mathrm{Li}$ for $\mathbf{q} \|$ [100] (NSLS data) is presented in Fig. 2 in comparison with a Lam-Platzman corrected ${ }^{18}$ LDA calculation ${ }^{8}$ without and with the SDF convolution. The experimental profile shows clear asymmetry and the Fermi breaks are significantly smeared compared with the LDA calculation. This is emphasized at the bottom panel of Fig. 2 which shows the second derivatives for the experiment and the theories, all convoluted with a Gaussian of 0.05 a.u. (FWHM) to reduce the numerical noise. One can clearly see that the major part of the observed extra broadening can be attributed to final-state effects due to the convolution according to Eq. (5) with the Lorentzian shaped

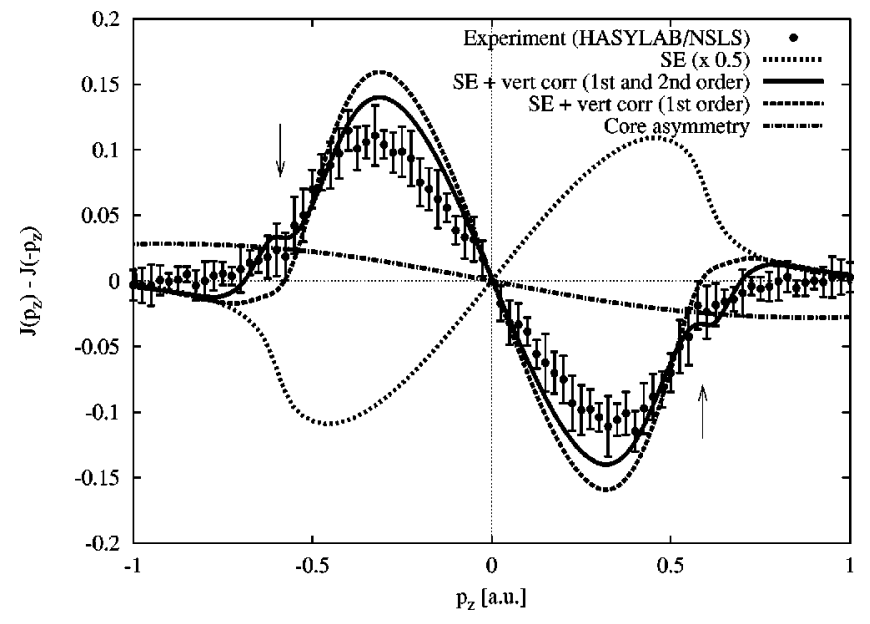

FIG. 3. Valence CP asymmetry compared to the calculation taking into account only the full SE correction (short dashed line) and in addition the first (long dashed line) and the first together with the second order vertex correction (solid line). The jellium $p_{F}$ is indicated by the arrows and the core asymmetry is plotted as dashed dotted line.

particle SDF, the FWHM of which is $\approx 0.07$ a.u. in strong contrast to an estimate in Ref. 6, which would result in a FWHM of $\approx 10^{-4}$ a.u. Nevertheless, an additional smearing of the Fermi break remains as found in $\mathrm{CP}$ measurements at higher incident energies. ${ }^{7,8}$ We have made a careful analysis also for the other directions with similar results.

The measured $\mathrm{CP}$ asymmetries can be separated into two different regimes: (i) For $\left|p_{z}\right|>p_{F}(=0.589$ a.u. for jellium) the core asymmetry dominates. The shape of the experimental asymmetry is in good agreement with the shape of the theoretical core asymmetry utilizing the first correction to the nonrelativistic Compton cross section in the IA. ${ }^{5}$ Deviations for $\left|p_{z}\right|>1.2$ a.u. (0.9 a.u. at NSLS) are due to the influence of the Li $K$ edge to the CP. (ii) For $\left|p_{z}\right|<p_{F}$ the shape of the total asymmetry changes rapidly due to the valence $\mathrm{CP}$ contribution. To extract the valence CP asymmetry for both experiments the core contribution (dashed dotted line in Fig. 3) was subtracted by fitting the shape of the theoretical core asymmetry to the experimental asymmetries in the range of 0.6 a.u. $<\left|p_{z}\right|<1.2$ a.u. (0.9 a.u.) where the amplitude of the core asymmetry was the fitting parameter. The asymmetries exhibit no significant differences concerning the direction of $\mathbf{q}$ within the experimental error and the experimental results achieved at HASYLAB and NSLS show good agreement which emphasizes the reliability of our experimental findings concerning the sign, the shape and even the amplitude of the valence $\mathrm{CP}$ asymmetry. Therefore, the average of the valence $\mathrm{CP}$ asymmetry over all directions of $\mathbf{q}$ and over the experiments is plotted in Fig. 3. The asymmetry of the calculated fully SE corrected PPF is shown as short dashed line and disagrees in sign and also in amplitude with the experiment. When the first order (long dashed line in Fig. 3) and the first together with the second order particle-hole vertex correction is added (solid line in Fig. 3), the sign of the asymmetry changes and one ends up with a good qualitative agreement between experiment and calculation which gives a strong indication for the incomplete cancellation of the SE correction and the vertex correction. We estimate that the 
third order vertex correction will contribute to the asymmetry by less than the experimental error. This way the $\mathrm{CP}$ asymmetry is a direct evidence of the electron-hole interaction in the course of the Compton scattering process. The parameter $\omega_{s}$ was estimated to $-3.5 \mathrm{eV}$ by fitting the energy positions of the Fermi breaks of the calculated CP's to the experimental peak positions of the second derivatives at $p_{F}$, which are symmetric with respect to $p_{z}=0$ (see Fig. 2) within the experimental error.

We have to conclude that the predicted sharp features of the Fermi break, which should be resolved with the well improved experimental resolution, are still smeared out. The additional broadening of the $\mathrm{CP}$ is attributed to the convolution of the CP with the excited particle SDF, which results in an intrinsic resolution limit for $\mathrm{CP}$ experiments performed at low incident energies. The Lorentzian contribution of the particle SDF to the momentum space resolution for $\mathrm{CP}$ measurements at 30 and $60 \mathrm{keV}$ incident photon energy is calculated to 0.009 and 0.002 a.u. FWHM, respectively, indicating that at higher energies the influence of the SDF becomes more and more negligible. This claims that the highly demanded improvement of the momentum space resolution be- yond 0.05 a.u. requires incident photon energies at least larger than $30 \mathrm{keV}$. Furthermore, the valence CP's exhibit an asymmetry which agrees in sign, shape and even amplitude to the predictions of the first and second order vertex correction to the fully SE corrected PPF. Therefore, it is clearly shown that the requirements of the IA are not fullfilled for $\mathrm{CP}$ measurements of valence electrons in the considered regime of incident energy and momentum transfer. The success of our many-particle calculation scheme supports its validity for a wide field of application ranging from final- state effects in photoemission and soft x-ray emission till to particle migration in solids.

This work was supported by the German Federal Ministry of Education and Research (05 ST8 HRA), the Academy of Finland (7379/40732), the DAAD (313/SF-PPP-PZ/ 9724260) and the U.S. Department of Energy (DE-AC0276CH00016). We have to thank A. Stewart for the $\mathrm{Li}$ samples used at NSLS and A. Bansil for giving us the LDA calculations $^{8}$ in numerical form. R. Heise is thanked for valuable help with the measurements.
${ }^{1}$ Compton Scattering, edited by B. Williams (McGraw-Hill, New York, 1977).

${ }^{2}$ K. Hämäläinen, S. Manninen, C.-C. Kao, W. Caliebe, J. B. Hastings, A. Bansil, S. Kaprzyk, and P. M. Platzman, Phys. Rev. B 54, 5453 (1996).

${ }^{3}$ P. Eisenberger and P. M. Platzman, Phys. Rev. B 6, 3671 (1970).

${ }^{4}$ A. Issolah, B. Levy, A. Beswick, and G. Loupias, Phys. Rev. A 38, 4509 (1988).

${ }^{5}$ P. Holm and R. Ribberfors, Phys. Rev. A 40, 6251 (1989), and references therein.

${ }^{6}$ P. M. Platzman and N. Tzoar, Phys. Rev. 139, A410 (1965).

${ }^{7}$ W. Schülke, G. Stutz, F. Wohlert, and A. Kaprolat, Phys. Rev. B 54, 14381 (1996).

${ }^{8}$ Y. Sakurai, Y. Tanaka, A. Bansil, S. Kaprzyk, A. T. Stewart, Y. Nagashima, T. Hyodo, H. Kawata, and N. Shiotani, Phys. Rev. Lett. 74, 2252 (1995).

${ }^{9}$ C. Filippi and D. M. Ceperley, Phys. Rev. B 59, 7907 (1999), and references therein.

${ }^{10}$ F. Green, D. Neilson, and J. Szymanski, Phys. Rev. B 31, 2796 (1985).

${ }^{11}$ K. S. Singwi and M. P. Tosi, in Solid State Physics, edited by H. Ehrenreich, F. M Seitz, and D. Turnbull (Academic, New York, 1981), Vol. 36, p. 177.

${ }^{12}$ W. Schülke and H. Nagasawa, Nucl. Instrum. Methods 222, 203 (1984).

${ }^{13}$ C.-C. Kao, K. Hämäläinen, M. Krisch, D. P. Siddons, T. Oversluizen, and J. B. Hastings, Rev. Sci. Instrum. 66, 1699 (1995).

${ }^{14}$ L. Hedin, Phys. Rev. 139, A796 (1965).

${ }^{15}$ A. Holas, P. K. Aravind, and K. S. Singwi, Phys. Rev. B 20, 4912 (1979).

${ }^{16}$ B. I. Lundqvist, Phys. Kondens. Mater. 6, 193 (1967).

${ }^{17}$ The inclusion of solely these diagrams second order in Coulomb interaction is only exemplary to show their order of magnitude.

${ }^{18}$ L. Lam and P. M. Platzman, Phys. Rev. B 9, 5122, 5128 (1974). 